\title{
Vom Nobelpreis und anderen Auszeichnungen
}

\author{
Nils Hanssona, Hubert Steinke ${ }^{b}$ \\ a PD Dr., Institut für Geschichte, Theorie und Ethik der Medizin, Universität Düsseldorf \\ ${ }^{b}$ Prof. Dr. Dr., Institut für Medizingeschichte, Universität Bern
}

Auch letztes Jahr fand am 10. Dezember wieder die feierliche Zeremonie statt. Der schwedische König übergab in Stockholm die Nobelpreise für Physik, Chemie, Physiologie oder Medizin und Literatur. Gleichzeitig fand in Bern ein vom Institut für Medizingeschichte und der Heinrich-Heine-Universität Düsseldorf organisiertes Symposium statt, das sich kritisch mit der Frage nach der Geschichte und dem Nutzen von Preisen für die Forschung befasste. Einen offensichtlichen Nutzen hob der schwedische Botschafter in der Schweiz, Magnus Hartog-Holm, gleich zu Beginn in seinem Grusswort hervor: «Die Aura der Exzellenz rund um den Nobelpreis stärkt das Bild von Schweden als Wissenschafts- und Kulturnation.» Er verdeutlichte damit, wie sehr der Nobelpreis über alle anderen Auszeichnungen hinausragt. Schon seit seiner ersten Verleihung 1901 ist der Nobelpreis das Vorbild, an dem sich viele Wissenschaftspreise orientieren. Er steht für die nachträgliche Auszeichnung einer hervorragenden Leistung, zumeist gegen Ende einer Forscherkarriere. Doch auf welcher Grundlage wird entschieden? Das seit einigen Jahren geöffnete Archiv des Nobelkomitees in Solna, Schweden, ermöglicht es, das Auswahlprozedere und die Chancen der zahlreichen erfolgreichen und erfolglosen Kandidaten zu rekonstruieren. Wir schauen uns die Nobelpreisnominierungen einiger Schweizer Chirurgen an.

\section{Schweizer Kandidaten}

Nobelpreise für die Entwicklung chirurgischer Methoden sind relativ rar. Der erste Preisträger in diesem Feld war Theodor Kocher (1841-1917), der im Jahr 1909 den Preis «für seine Arbeiten zur Physiologie, Pathologie und Chirurgie der Schilddrüse» erhielt [1]. Schon vor Kocher sind Schweizer Chirurgen für den Preis vorgeschlagen worden, darunter der Basler Psychiater und Hirnchirurg Gottlieb Burckhardt (1836-1907) und der Genfer Jacques-Louis Reverdin (1842-1929). Der vermutlich erste nominierte Schweizer Chirurg war der Zürcher Rudolf Ulrich Krönlein (1847-1910). Er wurde 1902 vom Internisten Oscar Wyss (1840-1918) vorgeschlagen. Wyss (1894-1896 Rektor der Universität Zürich) war vom schwedischen Karolinska Institut eingeladen worden, der Preisjury würdige Personen zu präsentieren. Seine Wahl fiel neben Krönlein auch auf den Berliner Urologen James Israel (1848-1926) [2]. Ihm zufolge seien die beiden Kandidaten wichtige Pioniere der Nierenchirurgie. Die Nominierung Krönleins ist aus heutiger Perspektive interessant, da dieser heute eher für andere Leistungen bekannt ist, etwa für Appendizitis-Operationen. Zur Nierenchirurgie hatte er zum Zeitpunkt des Nominierungsbriefes nicht viel publiziert. Womöglich gab es andere Gründe für den Vorschlag. Als zentrale Referenz für die Bedeutung Krönleins hob Wyss eine Publikation hervor, die dessen Assistent Max Oskar Wyss (1874-1956), zugleich Sohn des Nominators Oscar Wyss, zusammengestellt hatte. Persönliche Beziehungen zwischen Nominator und Kandidat sind keine Seltenheit. Um nur ein weiteres Beispiel zu nennen: Ferdinand Sauerbruch (18751951), Ordinarius für Chirurgie in Zürich 1914-1918, wurde in der ersten Hälfte des 20. Jahrhunderts rund sechzig Mal nominiert, primär von Schülern oder engen Fakultätskollegen [3]. Den Preis hat er nie erhalten.

\section{Jules Gonin - starker Nobelpreisaspirant}

Wurden Krönlein und Sauerbruch fast ausschliesslich von Landsleuten nominiert, sah es bei dem Lausanner Ophthalmologen Jules Gonin (1870-1935) anders aus. Für Gonin wurden zwischen 1930 und 1935 mindestens 
zwölf Nominierungen von mehreren internationalen Forschern eingereicht [4]. Als Hauptleistung Gonins galt sein Behandlungskonzept der Netzhautablösung, das der Wiener Augenarzt Josef Meller (1874-1968) in seiner Nominierung vorsichtig lobte: "Am meisten schien mir Gonin [...] für seine Entdeckung der Netzhautabhebung durch operativen Verschluss des Netzhautrisses in Betracht zu kommen, doch ist die Angelegenheit noch zu jung, als dass man ein endgültiges Urtheil über den Wert schon jetzt mit Sicherheit abgeben könnte.» Schlagkräftiger formulierte der amerikanische Augenarzt Harry Searls Gradle (1883-1950) seine Stellungnahme 1935: "It is acknowledged today that between $40 \%$ and $50 \%$ of cases of detachment of the retina can be cured. This is all due to Gonin.»

Tatsächlich stufte das Nobelkomitee Gonin 1934 als preiswürdig ein, schliesslich wählte es dann aber George Whipple (1878-1976), George Minot (1885-1950) und William P. Murphy (1892-1987) "for their discoveries concerning liver therapy in cases of anaemia" aus. Ein Jahr später starb Gonin. Da der Nobelpreis nicht posthum verliehen wird, wurde seine erneute Nominierung 1935 nicht evaluiert. Vermutlich wusste Jules Gonin von seinen Nobelpreischancen. Der Preiszeremonie stand er angeblich skeptisch gegenüber: «This saves me from the tail coat, the boredom of the ceremony, and the Scandinavian December cold" [5]. Unabhängig davon steht Gonin noch immer für Exzellenz. Die Gonin-Medaille gilt heute als eine der renommiertesten Auszeichnungen in seinem medizinischen Fachgebiet. Auch sie wurde einmal als «Nobel Prize in Ophthalmology» bezeichnet!

\section{Netzwerke}

Die Beispiele zeigen, dass viele Faktoren auf das Auswahlprozedere einwirken. Von zentraler Bedeutung war und ist bei Preisen das Netzwerk. Beim Nobelpreis wird Forschungsleistung seit jeher durch Gutachten und Expertenmeinungen aus dem entsprechenden Fachgebiet beurteilt. Man will sichergehen, dass der Ausgezeichnete in seinem Fachgebiet auch wirklich als bedeutender Forscher anerkannt ist. Diese Absicherung birgt aber die Gefahr, diejenigen Personen auszuzeichnen, die ein besonders gutes Netzwerk aufgebaut haben. Wie Matthias Egger, der Präsident des Schweizerischen Nationalfonds, der neu den Marcel-BenoistPreis vergibt, an der Tagung erläuterte, geht man beim «Schweizer Nobelpreis» einen neuen Weg. Man verzichtet auf Gutachten und versucht in einem ausgeklügelten, teilweise messenden Verfahren, eine Beurteilung vorzunehmen, die nicht von Forscher-Netzwerken beeinflusst ist.

\section{Superforscher}

Abgesehen von der Frage des Auswahlprozederes wurde an der Tagung auch die grundsätzlichere Frage gestellt, inwiefern die Auszeichnung einzelner Forscher der heutigen Wissenschaftslandschaft entspricht. Flurin Condrau (Zürich) betonte, dass es adäquater wäre, Preise an Forschungsgruppen statt an einzelne Personen zu verteilen. Doch offenbar ist unsere Gesellschaft fasziniert davon, einzelne Personen auszuzeichnen, und möchte dies auch entsprechend inszenieren. Beispielhaft sind die seit 2012 von IT-Milliardären vergebenen Breakthrough-Preise in Physik, Life Sciences und Mathematik mit einem Preisgeld von je drei Millionen Dollar. Die Preise werden von Celebritys aus Film- und Showbusiness vergeben, in einem Anlass, der dem Modell der Oscarverleihungen nachempfunden ist. Der Mix von viel Geld, Unterhaltung und Präsentation von aussergewöhnlichen Forschern soll die Begeisterung für Wissenschaft (und vielleicht auch für die IT-Milliardäre) fördern.

\section{Preise und Hämorrhoiden}

Man kann sich fragen, ob die Auszeichnung bereits hoch erfolgreicher und vielfach ausgezeichneter Forscher der beste Weg ist, die Wissenschaften zu fördern. Unbestritten an der Tagung war, dass die wichtigsten Preise diejenigen für Nachwuchsforscher sind. Sie motivieren und können wirksame Sprungbretter sein. Geht die wissenschaftliche Laufbahn weiter, so wirken weitere Preise als kontinuierliches Schmiermittel der Karriere. Und selbst wer bisher leer ausgegangen ist, kann sich mit Billy Wilders Spruch trösten: «Auszeichnungen und Preise sind wie Hämorrhoiden - früher oder später kriegt sie jedes A...»

\section{Bildnachweis}

Briefmarke Jules Gonin: @ Sergei Nezhinskii | Dreamstime.com

\section{Literatur}

1 Tröhler U. Theodor Kochers Nobelpreis. Voraussetzungen, Bedingungen, Folgen. Ein Blick hinter die Kulissen. In: Steinke H, Wolff E, Schmid R, editors. Schnitte, Knoten und Netze: 100 Jahre Schweizerische Gesellschaft für Chirurgie. Zürich: Chronos; 2013. p. 87-110.

2 Moll FH, Halling T, Hansson N, Fangerau H. «Wenn Sie alle vergessen sein werden, wird der Name Israel noch leuchten». James Israel (1848-1926): Eine Karriere im Deutschen Kaiserreich und seine Nominierung für den Nobelpreis. Urologe. 2017;56(3):369-81. doi: 10.1007/s00120-017-0326-3.

3 Hansson N, Schagen U. «In Stockholm hatte man offenbar irgendwelche Gegenbewegung»-Ferdinand Sauerbruch (1875-1951) und der Nobelpreis. NTM. 2014;22(3):133-61.

4 Hansson N, Geerling G, Halling T, Fangerau H. Augen auf bei der Wahl des Forschungsgebiets: Nobelpreiskandidaten in der Ophthalmologie 1901-1960. Klin Monbl Augenheilkd. 2017 (https://doi.org/10.1055/s-0043-118601).

5 Arruga A. Little known aspects of Jules Gonin's life. Doc Ophtalmol. 1997;94(1-2):83-90. 